\title{
Polibotánica
}

POLIBETÁNICA

ISSN electrónico: 2395-9525

polibotanica@gmail.com

Instituto Politécnico Nacional

México

http:www.polibotanica.mx

\section{EFECTO DE EXTRACTOS CRUDOS \\ DE AJO (Allium sativum) SOBRE EL \\ DESARROLLO in vitro DE Aspergillus parasiticus Y Aspergillus niger.}

\section{EFFECT OF GARLIC EXTRACTS (Allium sativum) ON THE DEVELOPMENT in vitro OF Aspergillus parasiticus AND Aspergillus niger.}

Juárez-Segovia, K.G.; E.J. Díaz-Darcía, M.D. Méndez-López, M.S. Pina-Canseco, A.D. Pérez-Santiago, y M.A. Sánchez-Medina.

EFECTO DE EXTRACTOS CRUDOS DE AJO (Allium sativum) SOBRE EL DESARROLLO in vitro DE Aspergillus parasiticus Y Aspergillus niger.

EFFECT OF GARLIC EXTRACTS (Allium sativum) ON THE DEVELOPMENT in vitro OF Aspergillus parasiticus AND Aspergillus niger.

POLIBeTÁNICA

Instituto Politécnico Nacional
Núm. 47: 99-111 México. Enero 2019

DOI: $10.18387 /$ polibotanica.47.8 
EFECTO DE EXTRACTOS CRUDOS DE AJO (Allium sativum) SOBRE EL
DESARROLLO in vitro DE Aspergillus parasiticus Y Aspergillus niger.

\section{EFFECT OF GARLIC EXTRACTS (Allium sativum) ON THE DEVELOPMENT in vitro OF Aspergillus parasiticus AND Aspergillus niger.}

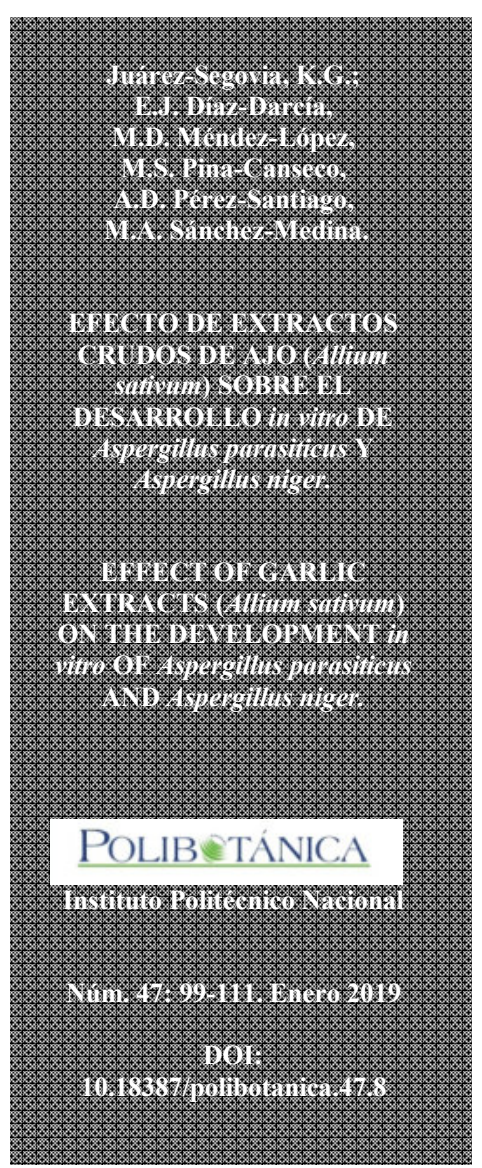

\begin{abstract}
K.G. Juárez-Segovia
E.J. Díaz-Darcía

M.D. Méndez-López

Laboratorio de alimentos, Departamento de Ingeniería Química y Bioquímica, Instituto Tecnológico de Oaxaca, Av. Victor Bravo Ahuja núm. 125 Esq. Calz. Tecnológico CP 68030, Oaxaca, Oaxaca, México.

M.S. Pina-Canseco Centro de Investigación Facultad de Medicina UNAM-UABJO, Facultad de Medicina y Cirugía, Universidad Autónoma "Benito Juárez” de Oaxaca, Ex Hacienda de Aguilera S/N, Carretera a San Felipe del Agua, CP 68020 Oaxaca, Oaxaca, México.

A.D. Pérez-Santiago Unidad de Bioquímica e Inmunología, División de Estudios de Posgrado e Investigación, Instituto Tecnológico de Oaxaca, Av. Víctor Bravo Ahuja núm. 125 Esq. Calz. Tecnológico CP 68030, Oaxaca, Oaxaca, México. M.A. Sánchez-Medina / marco.s.medina@itoaxaca.edu.mx
Laboratorio de alimentos, Departamento de Ingeniería Química y Bioquímica,
Instituto Tecnológico de Oaxaca, Av.Víctor Bravo Ahuja núm. 125
Esq. Calz. Tecnológico CP 68030, Oaxaca, Oaxaca, México.

RESUMEN: Aspergillus parasiticus y A. niger son dos hongos productores de micotoxinas; aflatoxinas y ocratoxinas respectivamente. Estas toxinas son causantes de enfermedades en seres humanos y animales, además de generar cuantiosas pérdidas económicas al contaminar cultivos de cereales, algodón y frutas secas entre otros. Como una alternativa para controlar el desarrollo de estos hongos, el objetivo del presente trabajo fue evaluar el efecto in vitro de extractos crudos de ajo sobre el desarrollo de Aspergillus parasiticus y $A$. niger mediante diferentes ensayos.

El extracto crudo de ajo (ECA) se preparó por maceración utilizando solución salina amortiguada por fosfatos (PBS) pH 7.2 como solvente. Se evaluó su efecto sobre el desarrollo de los hongos, lo cual se cuantificó mediante halos de inhibición, punción, unidades formadoras de colonias (UFC), determinación de la concentración mínima inhibitoria (CMI) y de la concentración fungicida. Adicionalmente se observó la interacción del extracto crudo de ajo con los hongos mediante microscopia de luz visible. El extracto crudo de ajo produjo halos de inhibición de $12 \mathrm{~mm}$ para A. parasiticus y de 15.5 mm para $A$. niger, e inhibió su crecimiento en un 13 y $46.8 \%$ respectivamente. La CMI para $A$. parasiticus se encontró en la dilución 1:2 (50 $\mu \mathrm{L}$ de extracto crudo) y para $A$. niger en la dilución 1:32 (3.12 $\mu \mathrm{L}$ de extracto crudo), y las concentraciones fungicidas se observaron en la dilución $1: 2(50 \mu \mathrm{L}$ de extracto crudo) y $1: 16(6.25 \mu \mathrm{L}$ de extracto crudo), respectivamente. Además, inhibió la producción de micelio y esporulación de los dos hongos.

El extracto crudo de ajo presentó actividad antifúngica frente a $A$. parasiticus y A. niger.
\end{abstract}


Palabras clave: Efecto antifúngico, Allium sativum (ajo), Aspergillus, aflatoxina, ocratoxina.

ABSTRACT: Aspergillus parasiticus and A. niger are two mycotoxin-producing fungi species, particularly aflatoxins and ochratoxins respectively. These toxins can cause diseases in humans and animals, in addition to substantial economic losses caused by the contaminating grain crops, cotton, dried fruits among other crops. In this study, we evaluate the effect of the garlic crude extract on the in vitro development of Aspergillus parasiticus and A. niger as a way to control the occurrence of these fungi on crops and other foods. The garlic crude extract (ECA) was elaborated by maceration using PBS pH 7.2 as solvent. The effect of the extract on the development of the fungi was evaluated by measuring of inhibition zones, puncture, colony forming units (CFU) and determination of minimum inhibitory concentration (MIC). The interaction of the garlic crude extract with the fungi was observed using visible light microscopy. The garlic crude extract induced inhibition zones of $12 \mathrm{~mm}$ in $A$. parasiticus and $15.5 \mathrm{~mm}$ for $A$. niger, it also inhibited growth in 13 and $46.8 \%$ respectively. The CMI for $A$. parasiticus was found to be the $1: 2$ dilution $(50 \mu \mathrm{L}$ of crude extract) and the 1:32 dilution (3.12 $\mu \mathrm{L}$ of crude extract) for $A$. niger. The treatment also inhibited the production of mycelium and sporulation of the two fungi especies.

The garlic crude extract showed antifungal activity against $A$. parasiticus and $A$. niger.

Key words: Antifungal effect, Allium sativum (ajo), Aspergillus, aflatoxin, ochratoxin.

\section{INTRODUCCIÓN}

Los hongos son los principales microrganismos causantes de la pérdida en la calidad de los cereales almacenados, además de ocasionar una disminución en la viabilidad o poder de germinación de la semilla, decoloración en el grano, pudrición, modificación del valor nutricional y características organolépticas, así como la producción de micotoxinas (Hernández, 2005; Pascual, Calderón, \& Calderón, 2000). Las micotoxinas son consideradas la principal causa de riesgo para la salud, ya que el consumo de granos contaminados, así como de sus productos puede ser fatal si estas se encuentran en altas concentraciones (Christensen \& López, 1962; Dohlman, 2004; Ocampo, 2006). Pueden ser producidas por algunos hongos del género Aspergillus y son de gran importancia debido a su alto potencial para producir enfermedades en humanos y animales, además de causar grandes pérdidas económicas. Como ejemplo de estas micotoxinas están las aflatoxinas producidas por A. flavus y $A$. parasiticus y las ocratoxinas producidas por A. niger (Juárez, 2002; Luna, Lozada, \& Trigos, 2010; Robledo, Marín, \& Ramos, 2001; Vega, 2012). Estos microorganismos son controlados con fungicidas tradicionales, lo cual podría causar graves consecuencias sobre la salud humana y el medio ambiente, además que algunos hongos han generado resistencia a ellos (Hernández, 2005). Una de las alternativas ha sido el uso de compuestos de origen vegetal, que tienen la habilidad de inhibir su crecimiento y/o la producción de sus micotoxinas (Gamboa, Hernández, Guerrero, Sánchez, \& Lira, 2003; Sánchez, 2002).

El ajo (Allium sativum) es un bulbo perteneciente a la familia Amaryllidaceae que se caracteriza por tener un sistema radicular constituido por una raíz bulbosa compuesta de 6 a 12 bulbillos, reunidos en su base por medio de una película delgada para formar la "cabeza del ajo" (Bender \& Bárcenas, 2013; Còrdova, 2010), ha sido utilizado por diversas civilizaciones en la elaboración de alimentos y en múltiples preparaciones medicinales y se considera que su origen se pudo haber dado en Asia Central y de ahí migrado a Arabia, Egipto, India, China y al Mediterráneo (Ledezma \& Apitz, 2006; Torija, Matallana, \& Chalup, 2013). El interés de la ciencia por la capacidad antifúngica del ajo se remonta al siglo pasado, Timonin y Thexton (1951) y Tansey (1975) observaron que extractos acuosos del ajo pueden inhibir el crecimiento de diversas especies de hongos. En la actualidad se conocen más de 100 compuestos biológicamente activos derivados de Allium sativum contenidos sobre todo en el bulbo. Destaca una sustancia sulfurada inodora llamada aliína que por acción de aliinasa se convierte en esencia de ajo y levulosa. La esencia de ajo contiene la alicina, a la cual se le atribuyen efectos antimicrobianos y antimicóticos in vitro, contra Candida albicans y algunos hongos, principalmente dermatofitos y levaduras patógenas para el hombre. Alkahil (2005) probó extractos acuosos, por arrastre de vapor y etanólicos de ajo (Allium sativum) contra Fusarium oxysporum, pero fue el extracto 
acuoso el mejor con casi 95\% de actividad fungicida (Corrales \& Reyes, 2016; Kyung, 2012; Ledezma \& Apitz, 2006; Moctezuma, Pedraza, Cárdenas, Martinez, \& Acosta, 2016; Villa et al., 2014). Este efecto está asociado a que los extractos de ajo disminuyen la absorción de oxígeno, reducen el crecimiento, dañan las membranas e inhiben la síntesis de lípidos, proteínas y ácidos nucleicos (Ankri \& Mirelman, 1999; Bender \& Bárcenas, 2013; Pundir \& Jain, 2010). El objetivo de este trabajo fue evaluar el efecto de extractos crudos de ajo sobre el desarrollo in vitro de $A$. parasiticus y A. niger mediante diferentes ensayos.

\section{MATERIAL Y MÉTODOS}

\section{Material vegetal}

Se utilizaron ajos (Allium sativum) obtenidos directamente del productor y cultivados entre enero y junio del 2017 en el municipio de San Jerónimo Tlacochahuaya, perteneciente al estado de Oaxaca, México.

\section{Preparación de extracto}

El extracto de ajo se preparó al momento de realizar los ensayos y se mantuvo en refrigeración entre 4 y $10^{\circ} \mathrm{C}$ hasta su uso. Los dientes de ajo fueron pelados y macerados a temperatura ambiente en un mortero de porcelana con solución salina amortiguada por fosfatos (PBS) pH 7.2 en relación 1:1 (p/v), esta solución se consideró la concentración al 100\%. Posteriormente el extracto se centrifugó por 10 min a $3000 \mathrm{rpm}$ para quitar los sólidos disueltos.

\section{Cepas fúngicas}

Los hongos utilizados fueron: A. parasiticus cepa ATCC 16992 donada por la doctora Dora Linda Guzmán Ortíz, Jefa del Laboratorio de Micotoxinas del CINVESTAV Unidad Irapuato y una cepa silvestre de $A$. niger, ambas conservadas en agua con tritón al $0.01 \%$.

\section{Preparación de las cepas}

Se inocularon $100 \mu \mathrm{L}$ de una suspensión de esporas en placas de PDA y se incubaron a $28 \pm 2{ }^{\circ} \mathrm{C}$ durante 7 días. Posteriormente, se recuperaron las esporas en agua con tritón al $0.01 \%$, se determinó su concentración con cámara de Neubauer y se ajustó a $1.5 \times 10^{6}$ esporas $/ \mathrm{mL}$.

\section{Evaluación in vitro de las propiedades antifúngicas del extracto de ajo}

Para evaluar las propiedades antifúngicas del extracto de Allium sativum se realizó mediante las Técnicas del pozo en agar y dilución de extracto en agar reportadas por Moreno, González, Salcedo, Cárdenas, \& Perales (2011) realizando modificaciones

\section{a) Técnica del pozo en agar}

Con espátula Drigalski se inocularon $100 \mu \mathrm{L}$ de la suspensión de esporas en placas de PDA, con un sacabocados se hicieron pozos de $5 \mathrm{~mm}$ de diámetro a los que se agregaron $10 \mu \mathrm{L}$ de extracto en diferentes concentraciones $(50,66,75,80$ y 100\%). Utilizando un control al cual se le agregaron $10 \mu \mathrm{L}$ de PBS en lugar del ECA. Las placas se incubaron a $28{ }^{ \pm} 2^{\circ} \mathrm{C}$ durante $72 \mathrm{~h}$, y los halos de inhibición se midieron cada $24 \mathrm{~h}$.

\section{b) Técnica de dilución del extracto en agar}

Debido a que en ensayos con concentraciones mayores a $1 \%$ de extracto crudo de ajo era imposible medir el efecto, se envenenó el medio PDA adicionando concentraciones de ECA por debajo de ese valor $(0.25,0.35,0.38$ y $0.4 \%)$. Se inoculó por punción en el centro de las placas con una colonia de $A$. parasiticus de $144 \mathrm{~h}$ de desarrollo, utilizando un control sin extracto. Las placas se incubaron durante 6 días a $28^{ \pm} 2^{\circ} \mathrm{C}$, y se midió el crecimiento radial de las colonias cada 24 horas. 
c) Unidades Formadoras de Colonias (UFC)

Para evaluar UFC se empleó la técnica reportada por Dìaz (2015), en tubos Eppendorf se agregaron $200 \mu \mathrm{L}$ de extracto en diferentes concentraciones $(50,66,75,80$ y $100 \%$ ) y $100 \mu \mathrm{L}$ de una suspensión de esporas $\left(1.5 \times 10^{3}\right.$ esporas $\left./ \mathrm{mL}\right)$, utilizando un control sin extracto. Los tubos se incubaron durante $48 \mathrm{~h}$ a $28^{ \pm} 2^{\circ} \mathrm{C}$. Posteriormente, el contenido de los tubos se inoculó por vertido en placas con PDA, se incubaron por $48 \mathrm{~h}$ a $28^{ \pm} 2^{\circ} \mathrm{C}$ y cada $24 \mathrm{~h}$ se realizó el conteo de colonias.

\section{Concentración Mínima Inhibitoria (CMI)}

Para determinar la concentración mínima inhibitoria se utilizó la técnica reportada por Moreno et al., (2011), realizando modificaciones, a partir de $100 \mu \mathrm{L}$ de extracto crudo, en tubos Eppendorf, se realizaron diluciones seriadas con $\operatorname{PBS}(1: 2,1: 4,1: 8,1: 16,1: 32,1: 64)$, posteriormente se agregaron $100 \mu \mathrm{L}$ de una suspensión de esporas $\left(1.5 \times 10^{6}\right.$ esporas $\left./ \mathrm{mL}\right)$ y $100 \mu \mathrm{L}$ de Caldo Papa Dextrosa (CPD); los tubos se incubaron $48 \mathrm{~h}$ a $28^{ \pm} 2^{\circ} \mathrm{C}$. Se realizó microscopía de luz visible para confirmar la ausencia de crecimiento micelial. Para evaluar el efecto fungicida del extracto de ajo sobre los hongos, los tubos que no presentaron crecimiento micelial visible en la prueba de CMI, se lavaron con agua destilada estéril por centrifugación y las esporas se resuspendieron en $100 \mu \mathrm{L}$ de agua destilada estéril, se inocularon en placas de PDA y se incubaron por seis días a $28^{\circ} \mathrm{C}+2^{\circ} \mathrm{C}$.

\section{Interacción del extracto crudo de ajo con los hongos}

Se realizó la misma técnica descrita para determinar la CMI y después de 48 h de incubación se realizó la observación por microscopia de luz visible para identificar los cambios morfológicos de esporas y micelio, el ensayo se realizó por triplicado.

\section{Análisis estadístico}

Se utilizó un diseño experimental complemente al azar y se realizó un análisis de varianza (ANOVA) de un solo factor. Además, se realizó una comparación de medias con la prueba de Tukey $(\mathrm{p}<0.05)$, utilizando el programa estadístico MINITAB (versión16).

\section{RESULTADOS Y DISCUSIÓN}

\section{Evaluación in vitro de las propiedades antifúngicas del extracto de ajo}

\section{a) Técnica del pozo en agar}

Los extractos crudos de ajo mostraron efecto inhibitorio sobre el crecimiento de $A$. parasiticus y $A$. niger, presentando halos de inhibición durante las 72 horas de incubación. El análisis de Tukey (p < 0.05) indicó que al cabo de 72 de incubación existe diferencia significativa entre las medias de los resultados del extracto al $100 \%$ y las concentraciones al 50, 66 y $75 \%$. Por otro lado, todas las concentraciones muestran diferencia significativa con respecto al control. El mayor efecto a las 72 horas de incubación lo presentó el extracto al 100\%, con halos de inhibición de $12 \mathrm{~mm}$ para $A$. parasiticus y $15.5 \mathrm{~mm}$ para $A$. niger (tabla 1), estos resultados son similares a los obtenidos con el extracto etanólico de propóleo y los extractos acuosos de canela y clavo al $100 \%$, los cuales presentaron halos de inhibición de $16,11.3$ y $15.4 \mathrm{~mm}$ respectivamente sobre A. niger (Bucio, Navarro, Martínez, \& Torres, 2016; Cubillo, 2007). En otro trabajo, el extracto acuoso de ajo en una concentración de $10 \mathrm{~g} / \mathrm{mL}$ presentó halos de inhibición de $0.5 \mathrm{~cm}$ sobre A. niger (Magro, Carolino, Bastos, \& Mexia, 2006). En ensayos realizados con $A$. flavus, hongo con características fisiológicas similares a $A$. parasiticus, el extracto de hoja de zimmu, un híbrido interespecífico de $A$. sativum y $A$. cepa, inhibió un $73 \%$ el crecimiento del hongo con respecto al control, presentando un halo de $2.4 \mathrm{~cm}$ (Sandosskumar et al., 2007). Con el mismo hongo, el extracto de Melissa officinalis en una concentración de $58.25 \mathrm{mg} / \mathrm{mL}$ presentó halos de inhibición de $22 \mathrm{~mm}$ (Abarca, Bragulat, Castellá, Accensi, \& Cabañes, 2000; Sara Centeno \& Carrera, 2013), mientras que los extractos etanólicos de Thymus vulgaris y Rosmarimus officinalis (S. Centeno, Calvo, Adelantado, \& Figueroa, 2010) produjeron halos de inhibición de $11.4 \mathrm{~mm}$ y $14.6 \mathrm{~mm}$ respectivamente, estos resultados son similares a los obtenidos en este trabajo con A. parasiticus. Algunos estudios han evaluado el efecto antifúngico de Allium sativum sobre otras especies de hongos. Un liofilizado de Allium sativum en una 
concentración de $400 \mu \mathrm{g} / \mathrm{mL}$ mostró halos de inhibición entre 1500-2000 mm para Trichophyton mentagrophytes, Trichophyton rubrum, Microsporum canis y Candida albicans (Lora, Lujan, Robles, Saravia, \& Cabeza, 2010). El extracto acuoso de Allium sativum a concentración de 9 y $10 \mathrm{~g} / \mathrm{ml}$ inhibió el desarrollo de Fusarium culmorum, Penicillium sp. y Aspergillus candidus, produciendo halos de inhibición de 0.5-0.7 cm (Magro et al., 2006). En otro estudio el extracto de ajo $(200 \mathrm{mg} / \mathrm{ml}) \mathrm{mostró}$ halos de $20 \mathrm{~mm}$ sobre Trichophyton rubrum (Samuel, Andrews, \& Jebashree, 2000).

Tabla 1. Valores promedio (mm) de los halos de inhibición de A. parasiticus y A. niger en respuesta a diferentes porcentajes de extracto crudo de ajo.

\begin{tabular}{|c|c|c|c|}
\hline \multirow{2}{*}{$\begin{array}{c}\text { Porcentaje de } \\
\text { extracto crudo }\end{array}$} & \multicolumn{3}{|c|}{ Halo de inhibición (mm *) } \\
\cline { 2 - 4 } & $\mathbf{3}$ & $\mathbf{3}$ & $\mathbf{4}$ A. parasiticus \\
\cline { 2 - 4 } & \multicolumn{3}{|c|}{ T2 $^{*}$} \\
\hline 50 & $14.3^{\mathrm{c}}$ & $9.5^{\mathrm{c}}$ & $5.3^{\mathrm{d}}$ \\
\hline 66 & $17.5^{\mathrm{b}, \mathrm{c}}$ & $14.2^{\mathrm{b}}$ & $6^{\mathrm{c}, \mathrm{d}}$ \\
\hline 75 & $20^{\mathrm{a}, \mathrm{b}}$ & $17.7^{\mathrm{a}, \mathrm{b}}$ & $8.6^{\mathrm{b}, \mathrm{c}}$ \\
\hline 80 & $20.5^{\mathrm{a}, \mathrm{b}}$ & $17.9^{\mathrm{a}}$ & $9.8^{\mathrm{a}, \mathrm{b}}$ \\
\hline 100 & $22.4^{\mathrm{a}}$ & $19.5^{\mathrm{a}}$ & $12^{\mathrm{a}}$ \\
\hline Control & $0^{\mathrm{d}}$ & $0^{\mathrm{d}}$ & $0^{\mathrm{e}}$ \\
\hline & & \multicolumn{3}{|c|}{ niger } \\
\hline 50 & $30.1^{\mathrm{a}}$ & $19.1^{\mathrm{a}}$ & $11^{\mathrm{a}}$ \\
\hline 66 & $30.8^{\mathrm{a}}$ & $19.8^{\mathrm{a}}$ & $12.3^{\mathrm{a}, \mathrm{b}}$ \\
\hline 75 & $31.5^{\mathrm{a}}$ & $20.3^{\mathrm{a}}$ & $13.6^{\mathrm{a}, \mathrm{b}}$ \\
\hline 80 & $31.7^{\mathrm{a}}$ & $20.8^{\mathrm{a}}$ & $13.8^{\mathrm{b}, \mathrm{c}}$ \\
\hline 100 & $31.9^{\mathrm{a}}$ & $25.5^{\mathrm{b}}$ & $15.5^{\mathrm{c}}$ \\
\hline Control & $0^{\mathrm{b}}$ & $0^{\mathrm{c}}$ & $0^{\mathrm{d}}$ \\
\hline
\end{tabular}

*El halo de inhibición incluye el diámetro del pozo $(5 \mathrm{~mm})$; Los resultados son la media de seis repeticiones.

**Letras diferentes en la columna indican diferencias significativas (Tukey, $\mathrm{p}<0.05$ )

b) Técnica de dilución del extracto en agar.

En los ensayos de dilución del extracto en agar, El análisis de Tukey $(\mathrm{p}<0.05)$ indicó que al cabo de 144 de incubación existe diferencia significativa entre las medias de los resultados del extracto al 0.38 y $0.4 \%$ con respecto al control de $A$. parasiticus (tabla 2), el crecimiento disminuyó en 10 y $13 \%$ con colonias de 69.6 y $66.8 \mathrm{~mm}$ respectivamente, después de 144 horas de incubación. Con $A$. niger, el extracto de ajo mostró una diferencia significativa en todas las concentraciones con respecto al control. La concentración que inhibió en mayor porcentaje el crecimiento del hongo fue la de $0.4 \%$ con un $46.8 \%$ con una colonia de $40.1 \mathrm{~mm}$ (tabla 2 ).

Por otro lado, algunos estudios reportan que los extractos de canela y orégano en concentración de 250 ppm y los extractos etanólicos de Larrea tridentata, en una concentración de $100 \mathrm{mg} / \mathrm{mL}$ inhibieron en un $100 \%$ el crecimiento de A. flavus (Moreno et al., 2011), los extractos etanólicos de Allium sativum y Allium cepa a una concentración de $1 \mathrm{~g} / \mathrm{mL}$ después de siete días de incubación inhibieron el desarrollo de A. flavus en 56.25 y $37.5 \%$ respectivamente (Tagoe, Nyarko, \& Akpaka, 2011). Los extractos de Juniperus sabina y J. virginiana en concentraciones de $1000 \mathrm{ppm}$ inhibieron el crecimiento de $A$. parasiticus en un 100\% (Dambolena et al., 2011; García et al., 2006). Los extractos metanólicos de Karwinskia humboldtiana y Ambrosia ambrosioides al 6\% inhibieron el crecimiento de A. niger en un 52 y $50 \%$ respectivamente, mientras que los extractos etanólicos de L. tridentata 
inhibieron el crecimiento de la misma especie en un 77.3\% (Tequida, Cortez, Rosas, López, \& Corrales, 2002); estos resultados son similares a los observados en este trabajo con el extracto crudo de ajo. Por otra parte, extractos metanólicos de Myrocaroues frondosus inhibieron en un 17\% el crecimiento micelial de A. niger, los extractos etanólicos de Allium sativum y Allium cepa a una concentración de $1 \mathrm{~g} / \mathrm{mL}$ después de siete días de incubación inhibieron en un 46.2 y $30.8 \%$ el desarrollo de A. niger (Jerke et al., 2008; Tagoe et al., 2011; Torija et al., 2013), porcentaje menor al observado con el extracto crudo de ajo. Por otro lado, el extracto acuoso de Allium sativum a una concentración de $100 \mathrm{mg} / \mathrm{ml}$ inhibió en un $100 \%$ el desarrollo de Trichophyton rubrum (Samuel et al., 2000).

Tabla 2. Valores promedio (mm) del crecimiento radial de A. parasiticus y A. niger en respuesta a diferentes porcentajes de extracto crudo de ajo.

\begin{tabular}{|c|c|c|c|c|c|c|}
\hline \multirow{4}{*}{$\begin{array}{c}\text { Concentración } \\
\text { (ppm) }\end{array}$} & \multicolumn{6}{|c|}{ Crecimiento radial $\left(\mathrm{mm}^{*}\right)$} \\
\hline & \multicolumn{6}{|c|}{ Tiempo de incubación (h) } \\
\hline & 24 & 48 & 72 & 96 & 120 & 144 \\
\hline & \multicolumn{6}{|c|}{ A. parasiticus } \\
\hline 0.25 & $4^{\mathrm{a}}$ & $16.8^{\mathrm{a}}$ & $30^{\mathrm{a}, \mathrm{b}}$ & $42.1^{\mathrm{a}}$ & $55^{\mathrm{a}}$ & $76.1^{\mathrm{a}}$ \\
\hline 0.35 & $3^{\mathrm{a}}$ & $15.8^{\mathrm{a}}$ & $29.6^{\mathrm{a}, \mathrm{b}}$ & $41.8^{\mathrm{a}}$ & $50.6^{\mathrm{b}}$ & $74^{\mathrm{a}}$ \\
\hline 0.38 & $1.5^{\mathrm{b}}$ & $13^{b, c}$ & $26.6^{\mathrm{b}, \mathrm{c}}$ & $38.2^{\mathrm{a}, \mathrm{b}}$ & $50^{\mathrm{b}, \mathrm{c}}$ & $69.6^{b}$ \\
\hline 0.4 & $1.1^{\mathrm{b}}$ & $12^{\mathrm{c}}$ & $26.1^{\mathrm{c}}$ & $37.1^{\mathrm{b}}$ & $48.8^{\mathrm{c}}$ & $66.8^{\mathrm{b}}$ \\
\hline \multirow[t]{2}{*}{ Control } & $6.6^{\mathrm{c}}$ & $18.6^{\mathrm{a}}$ & $32.6^{\mathrm{a}}$ & $47.1^{\mathrm{c}}$ & $59.5^{\mathrm{d}}$ & $77.5^{\mathrm{a}}$ \\
\hline & \multicolumn{6}{|c|}{ A. niger } \\
\hline 0.25 & $2.3^{\mathrm{b}}$ & $6.3^{\mathrm{b}}$ & $21.5^{\mathrm{b}}$ & $35.2^{\mathrm{b}}$ & $47.6^{\mathrm{b}}$ & $56.8^{\mathrm{b}}$ \\
\hline 0.35 & $0^{c}$ & $5.5^{\mathrm{b}}$ & $16.2^{\mathrm{b}, \mathrm{c}}$ & $26.2^{\mathrm{c}}$ & $40.2^{b}$ & $51.8^{\mathrm{c}}$ \\
\hline 0.38 & $0^{c}$ & $3.2^{\mathrm{c}}$ & $10.8^{\mathrm{c}}$ & $21.5^{\mathrm{d}}$ & $39.8^{\mathrm{c}}$ & $51.7^{\mathrm{c}}$ \\
\hline 0.4 & $0^{c}$ & $2.3^{\mathrm{c}}$ & $9.1^{\mathrm{c}}$ & $21.5^{\mathrm{d}}$ & $35.8^{\mathrm{d}}$ & $40.1^{\mathrm{d}}$ \\
\hline Control & $10.2^{\mathrm{a}}$ & $27.6^{\mathrm{a}}$ & $42^{\mathrm{a}}$ & $62.7^{\mathrm{a}}$ & $68.3^{\mathrm{a}}$ & $75.3^{\mathrm{a}}$ \\
\hline
\end{tabular}

*Letras diferentes en la columna indican diferencias significativas (Tukey, $\mathrm{p}<0.05$ )

**En el cuadro se muestra la media de seis repeticiones.

\section{c) Unidades formadoras de colonias (UFC)}

Para evaluar la viabilidad de las esporas se realizó la prueba de UFC. Para A. parasiticus y A. niger, todas las concentraciones mostraron una inhibición del $100 \%$ con respecto al control sin extracto (figs. 1 y 2). En un estudio similar, Díaz (2013), reportó que el extracto de lectina de coleoptilo de Zea mays, incrementaba el número de colonias de A. parasiticus con respecto al control sin lectina, y que el extracto crudo de Sorghum bicolor a mayor concentración disminuía el desarrollo de sus colonias.

\section{Concentración mínima inhibitoria (CMI)}

En la prueba para determinar la CMI, la dilución 1:2, que corresponde a $50 \mu \mathrm{L}$ de extracto crudo, inhibió en $100 \%$ el crecimiento de $A$. parasiticus. En un trabajo similar, la CMI para $A$. flavus del extracto de Melissa officinalis se observó en la dilución 1:2.5 $(23.30 \mathrm{mg} / \mathrm{mL})$ y en el extracto etanólico de ajo en $20 \mathrm{mg} / \mathrm{mL}$ (Sara Centeno \& Carrera, 2013; Pundir \& Jain, 2010). Para A. niger, la CMI se observó en la dilución 1:32 (3.12 $\mu \mathrm{L}$ de extracto). Al respecto, Huamanì \& Ruiz (2005), reportaron una CMI de $4 \mathrm{mg} / \mathrm{mL}$ con los extractos de Annona para este hongo. En trabajos realizados con extractos de ajo se encontró que la concentración mínima inhibitoria (CMI) del extracto concentrado fresco del ajo (ECFA) estaba entre 40 y $50 \mu \mathrm{L}$ del ECFA $(0.8-1.0 \mathrm{mg} / \mathrm{mL}$ de proteína) sobre los hongos Histoplasma capsulatum, Cladophialophora carrionii y Exophiala dermatitidis (Moctezuma Zárate et al., 2016). 

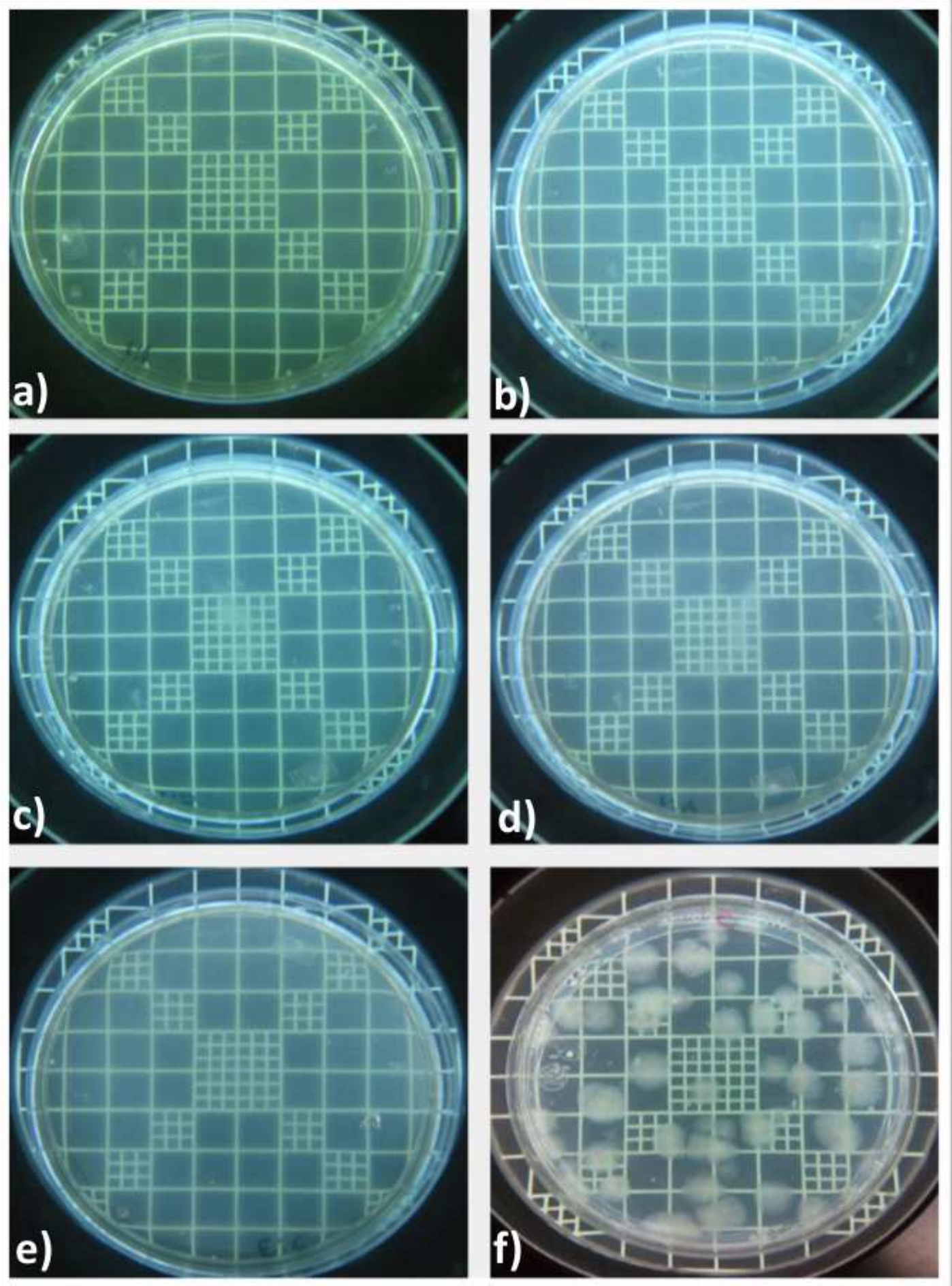

Fig. 1. Unidades Formadoras de Colonias de A. parasiticus después de 48 horas de incubación con extractos de ajo al a) $50 \%$, b) $66 \%$, c) $75 \%$, d) $80 \%$, e) $100 \%$ y f) control sin extracto. 

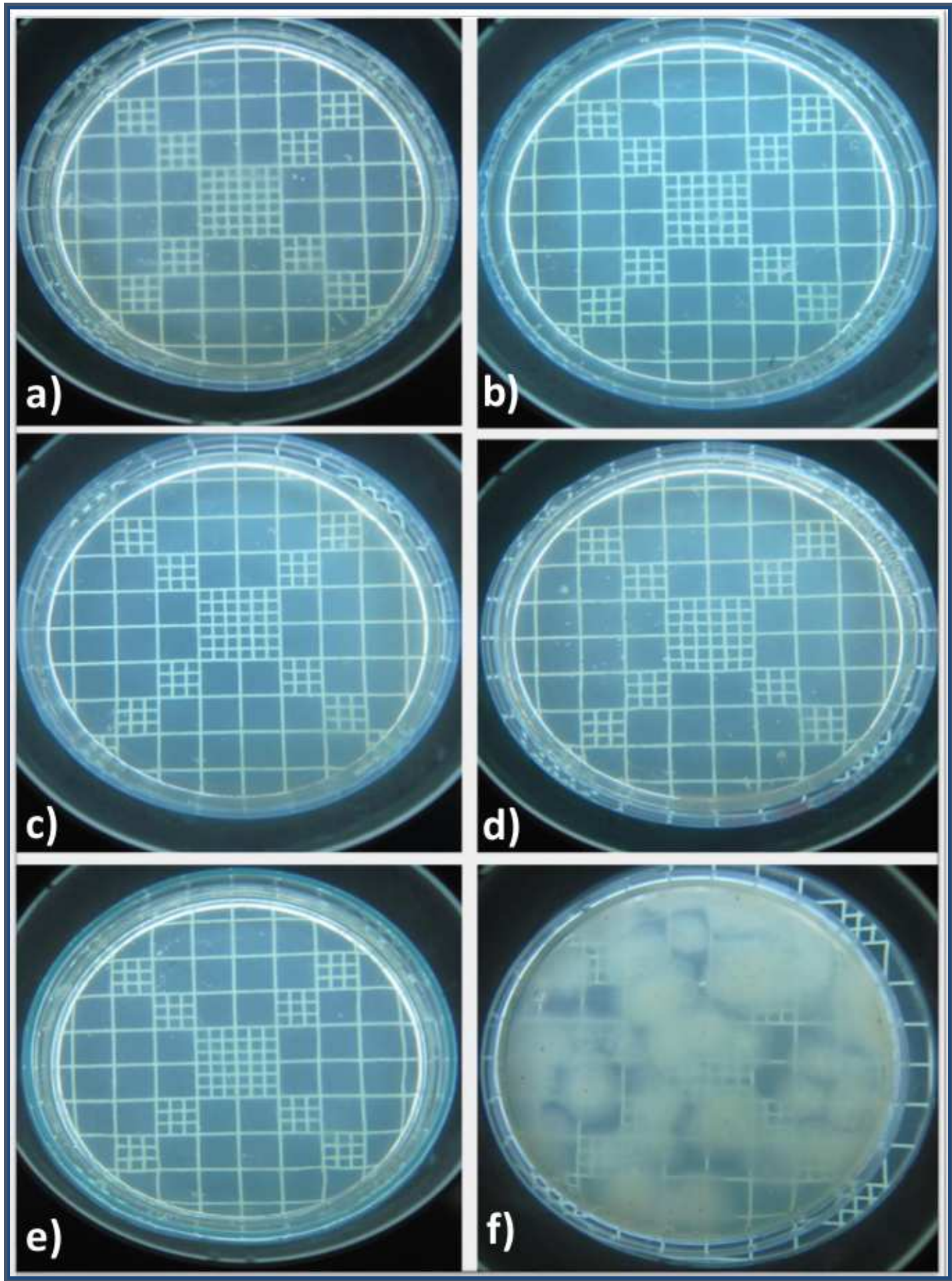

Fig. 2. Unidades Formadoras de Colonias de A. niger después de 48 horas de incubación con extractos de ajo al a) 50\%, a) $66 \%$, c) $75 \%$, d) $80 \%$, e) $100 \%$ y f) control sin extracto. 
Allium sativum presentó una CMI a una concentración de $500 \mu \mathrm{g} / \mathrm{mL}$ sobre Microsporum canis, Trichophyton mentagrophytes y Trichophyton rubrum, y de $2500 \mu \mathrm{g} / \mathrm{mL}$ sobre Candida albican (Lora et al., 2010). El compuesto activo del ajo, ajoeno presento una CMI en medio CSD de 2.5 y 5 en medio RPMI-1640, de 1,25 a $5 \mu \mathrm{g} / \mathrm{ml}$ sobre Histoplasma capsulatum (Torres \& Romero, 2012).

Para A. parasiticus la concentración fungicida se encontró en la dilución 1:2, correspondiente a $50 \mu \mathrm{L}$ de extracto de ajo, y para $A$. niger la concentración fungicida se observó en la dilución $1: 16,(6.25 \mu \mathrm{L}$ de extracto). García et al. (2006), reportaron concentraciones fungicidas de 1000 y 2000 ppm, sobre $A$. flavus con los extractos de orégano y canela respectivamente. Allium sativum liofilizado presentó una concentración fungicida de $1000 \mu \mathrm{g} / \mathrm{mL}$ sobre Histoplasma capsulatum, Cladophialophora carrionii y Exophiala dermatitidis, y de $5000 \mu \mathrm{g} / \mathrm{mL}$ para Candida albicans, por otro lado el ajoeno tiene una concentración fungicida de 5 y $10 \mu \mathrm{g} / \mathrm{ml}$ sobre Histoplasma capsulatum (Lora et al., 2010; Torres \& Romero, 2012).

\section{Interacción del extracto crudo de ajo con los hongos}

En los estudios microscópicos se observó que el extracto crudo de ajo inhibió el crecimiento micelial de los dos hongos en la dilución 1:32, y redujo significativamente la cantidad de esporas, en comparación con el control sin extracto, en el cual se observó elongación de hifas y esporulación de cabezas conidiales, por lo que se puede concluir que el extracto crudo de ajo inhibe la germinación de las esporas de A. parasiticus y A. niger (fig. 3). Lo cual es diferente a lo reportado para las lectinas Concavalina A y Dolichos biflorus sobre A. parasiticus, las cuales indujeron la elongación de hifas y modificaron la morfología de las mismas (Dìaz, 2015).

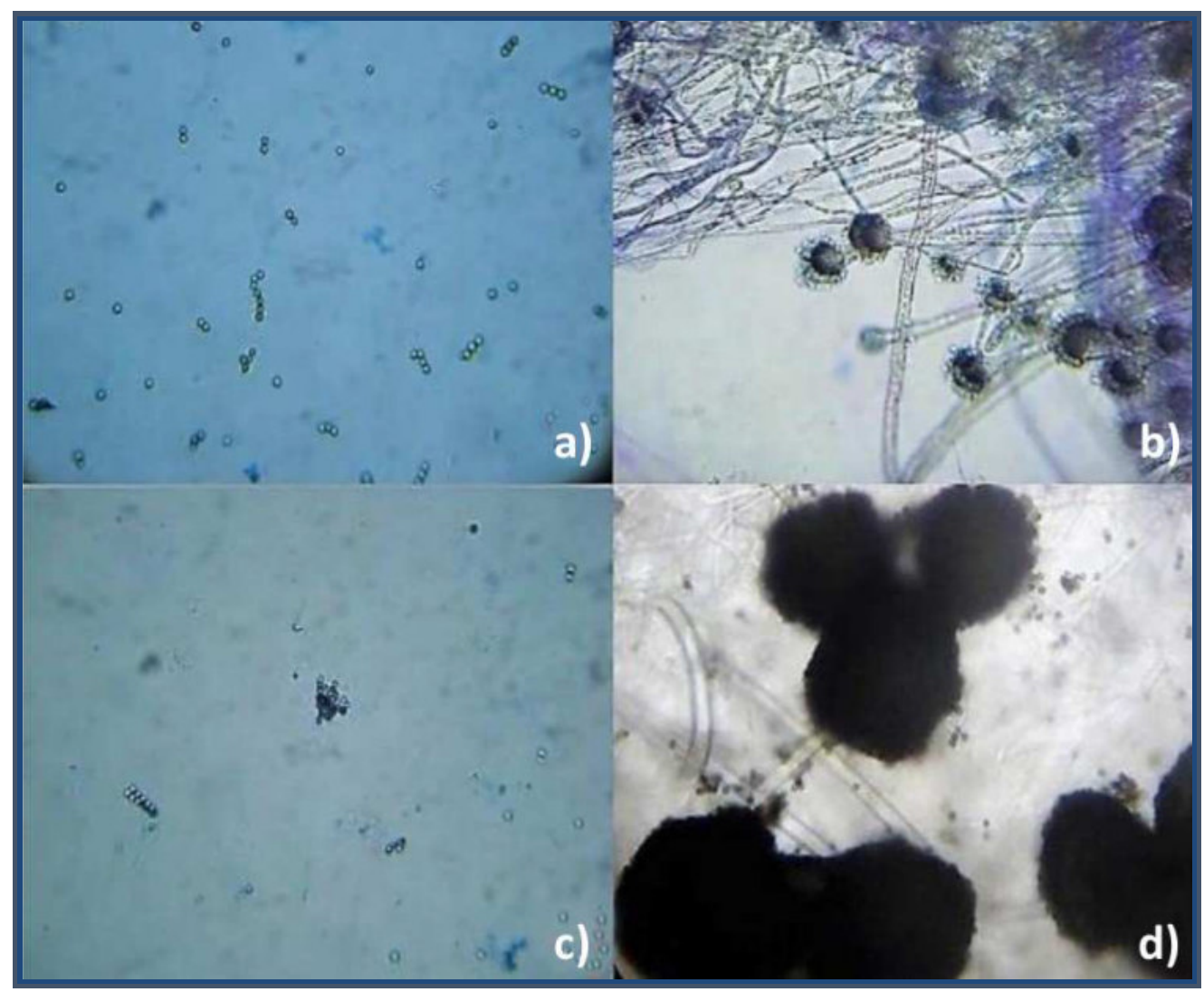

Fig. 3. Microscopia de la interacción del extracto crudo de ajo con los hongos, Vista 40x: a) ECA- $A$. parasiticus, b) Control de $A$. parasiticus, c) ECA-A. niger, d) Control de $A$. niger. 
Se ha reportado que algunos extractos vegetales contienen sustancias que muestran un efecto directo en la reducción o total inhibición del crecimiento de Aspergillus, mediante la degradación de componentes estructurales de pared celular, membrana celular, y organelos (Bhatnagar, Cary, Ehrlich, Yu, \& Cleveland, 2006; Cupull, Andreu, Pérez, Delgado, \& Cuppull, 2003). Y con respecto al extracto concentrado de ajo, la inhibición del crecimiento bacteriano se atribuye principalmente a la alicina (Jiménez \& Zambrano, 2017; Moctezuma Zárate et al., 2016). Su efecto radica en el bloqueo de la síntesis de fosfatidilcolina, con una acumulación del compuesto precursor fosfatidiletanolamina, lo cual se traduce en una alteración en la estructura de la membrana celular y consecuente muerte celular (San Blas et al., 1997).

\section{CONCLUSIONES}

Allium sativum inhibe el crecimiento de A. flavus y A. niger in vitro. Debido a que se trabajó con un extracto acuoso, se puede atribuir el efecto antifúngico a la presencia de alicina; se presentó un mayor efecto sobre $A$. niger en algunos de los ensayos. La concentración mínima inhibitoria para $A$. parasiticus se encontró en la dilución 1:2 y para $A$. niger en 1:32, y las concentraciones fungicidas se observaron en las concentraciones 1:2 y 1:16 respectivamente. Se demostró mediante microscopia que a mayor concentración de extracto crudo de ajo mayor inhibición de la producción de micelio y esporulación en comparación con el control. En general, el efecto de los extractos de ajo dependerá de su concentración, volumen, tipo de extracción y la especie de hongo a estudiar.

\section{Agradecimientos}

Al Consejo Nacional de Ciencia y Tecnología (CONACYT) por la beca para estudios de doctorado. Parte este trabajo se realizó con financiamiento del Tecnológico Nacional de México a través del proyecto 5814.16-P.

\section{LITERATURA CITADA}

Abarca, L., Bragulat, R., Castellá, G., Accensi, F., \& Cabañes, J. (2000). Hongos Productores de Micotoxinas Emergentes. Revista Iberoamericana de Micología, 17, 63-68.

Ankri, S., \& Mirelman, D. (1999). Antimicrobial Properties of Allicin from Garlic. Microbes and Infection, 2, 125-129.

Bender, D., \& Bárcenas, M. E. (2013). El ajo y sus Aplicaciones en la Conservación de Alimentos. Temas Selectos de Ingeniería de Alimentos, 7, 25-36.

Bhatnagar, D., Cary, J., Ehrlich, K., Yu, J., \& Cleveland, T. (2006). Understanding the Genetics of Regulation of Aflatoxin Production and Aspergillus flavus Development. Mycopathologia, 162, 155-166.

Bucio, C. M., Navarro, F. A., Martínez, O. A., \& Torres, J. J. (2016). Efecto sobre Hongos Fitopatógenos de un Extracto Acuosos de Propóleo Obtenido de las Abejas Domésticas. Investigación y Desarrollo en Ciencia y Tecnología de Alimentos, 1(2), 107-110.

Centeno, S., Calvo, M. A., Adelantado, C., \& Figueroa, S. (2010). Antifungal Activity of Extracts of Rosmarinus officinalis and Thymus vulgaris against Aspergillus flavus and A. ochraceus. Pakistan Journal of Biological Sciences, 13(9), 452-455.

Centeno, S., \& Carrera, Y. (2013). Actividad Antifúngica y Antiaflatoxigénica de Extractos de Melissa officinalis (Lamiaceae) sobre Aspergillus flavus. Saber, 25(2), 185-191.

Christensen, C., \& López, L. (1962). Daños Que Causan en México los Hongos de Granos Almacenados. (Vol. 44). Ciudad de México.

Còrdova, M. de los Á. (2010). Extracción y Purificación de Alicina a partir de Ajo (Allium sativum L): Implicaciones Analíticas. Instituto Politécnico Nacional. 
Corrales, I., \& Reyes, J. (2016). Actividad Antimicrobiana y Antifúngica de Allium sativum en Estomatología. 16 de Abril, (254), 59-68. Recuperado de https://www.researchgate.net/publication/308201470

Cubillo, M. A. (2007). Determinación de la Actividad Antimicrobiana de Algunas Especies Naturales sobre Microorganismos Asociados a Alimentos. Universidad de Costa Rica.

Cupull, R., Andreu, C., Pérez, C., Delgado, Y., \& Cuppull, M. (2003). Efecto de Trichoderma viride como Estimulante de la Germinación, en el Desarrollo de Posturas de Cafetos y el Control de Rhizoctonia solani Kuhn. Centro Agrícola, (1), 21-25.

Dambolena, J. S., Meriles, J. M., López, A. G., Gallucci, M. N., González, S. B., Guerra, A., \& Zunino, M. (2011). Actividad Antifúngica del Aceite Esencial de Cinco Especies de Juniperus de Argentina. Boletín Latinoamericano y del Caribe de Plantas Medicinales y Aromáticas, 10(2), 104-115.

Díaz, E. (2013). Interacción de las Lectinas de Zea mays y Sorghum bicolor con el Hongo Aspergillus parasiticus. Instituto Tecnológico de Oaxaca.

Dìaz, E. (2015). Evaluación del Efecto de la Interacción de las Lectinas Concavalina A y Dolichos biflorus con Aspergillus parasiticus. Instituto Tecnológico de Oaxaca.

Dohlman, E. (2004). Mycotoxin Regulations Implications for International Agricultural Trade. Agriculture Information Bulletin.

Gamboa, R., Hernández, F., Guerrero, E., Sánchez, A., \& Lira, R. (2003). Inhibición del Crecimiento Micelial de Rhizoctonia solani Kuhn y Phytophthora infestans Mont. (De Bary) con Extractos Vegetales Metanólicos de Hojasén (Flourensia cernua D.C.), Mejorana (Origanum majorana L.) y Trompetilla [Bouvardia ternifolia (Ca) Schlech]. Revista Mexicana de Fitopatología, 21(1), 13-18.

García, A. E., Quezada, Y. M., Moreno, J., Sánchez, G., Moreno, E., \& Pérez, M. C. (2006). Actividad Antifúngica de Aceites Esenciales de Canela (Cinnamomum zeylanicum Blume) y Orégano (Origanum vulgare L.) y su Efecto sobre la Producción de Aflatoxinas en Nuez Pecanera [Carya illinoensis (F.A. Wangenh) K. Koch]. Revista Mexicana de Fitopatología, 24(1), 8-12.

Hernández, M. (2005). Efectividad Antibacterial y Antifúngica de Quitosan y Larrea tridentata contra Microorganismos que Afectan a Humanos y Productos Agrícolas. Universidad de Guadalajara.

Huamanì, M. ., \& Ruiz, J. (2005). Determinación de la Actividad Antifùngica contra Candida albicans y Aspergillus niger de 10 plantas Medicinales de 3 Departamentos del Perú. Universidad Nacional Mayor de San Marcos.

Jerke, G., Horianski, M., Bargarbi, S., Salvatierra, K., Kramer, F., Jorda, G., ... Guida, A. (2008). Actividad Antifúngica de Extractos de Myrocarpus frondosus Fr. Allem sobre Hongos Aislados de Yerba mate y Té Comerciales. Ciencia y Tecnología, 10a, 24-29.

Jiménez, A., \& Zambrano, M. (2017). Efecto Antibacteriano del Extracto de Allium sativum (Ajo) Blanco, Púrpura y Clorhexidina al $0.12 \%$ sobre Cepas de Streptococcus mutans. Dominio de las Ciencias, 3(1), 234-247.

Juárez, S. R. (2002). Extractos de Larrea tridentata con Actividad Antifúngica e Inhibición de la Síntesis de Aflatoxinas de Especies del Género Aspergillus. Universidad Autónoma de Nuevo León.

Kyung, K. H. (2012). Antimicrobial Properties of Allium Species. Current Opinion in Biotechnology, 23, 142-147. https://doi.org/10.1016/j.copbio.2011.08.004

Ledezma, E., \& Apitz, R. (2006). Ajoene, el Principal Compuesto Activo Derivado del Ajo (Allium sativum), un Nuevo Agente Antifúngico. Revista Iberoamericana de Micología, 23, 75-80.

Lora, C., Lujan, M., Robles, H., Saravia, V., \& Cabeza, J. (2010). Efecto in vitro de Diferentes Concentraciones de Allium sativum (Ajo) Frente a Dermatofitos y Candida albicans. UCV-Scientia, 2(2), 23-33.

Luna, M., Lozada, Y., \& Trigos, Á. (2010). Aislamiento de Cepas de Aspergillus niger, Productoras de Ocratoxina A, en Café Verde (Coffea arabica) Almacenado. Revista Mexicana de Micología, 32, 64-68. 
Recibido:

19/mayo/2018

Aceptado: 10/diciembre/2018
Magro, A., Carolino, M., Bastos, M., \& Mexia, A. (2006). Efficacy of Plant Extracts Against Stored Products Fungi. Revista Iberoamericana de Micologia, 23, 176-178.

Moctezuma Zárate, M., Pedraza Ramos, M., Cárdenas Gonzáles, J., Martinez Juárez, V., \& Acosta Rodriguez, J. (2016). Efecto del ajo (Allium sativum) sobre el crecimiento de algunas especies de hongos. Tlatemoani, Revista Académica de Investigación.

Moreno, S., González, L. M., Salcedo, S. M., Cárdenas, M. L., \& Perales, A. (2011). Efecto Antifúngico de Extractos de Gobernadora (Larrea tridentata L.) sobre la Inhibición In vitro de Aspergillus flavus y Penicillium SP. Polibotánica, (32), 193-205.

Ocampo, I. (2006). Estudio de la Micoflora y Contenido de Aflatoxinas de Cebada Cultivada en Tlanalapa, Hidalgo. Universidad Autónoma del Estado de Hidalgo.

Pascual, M. R., Calderón, V., \& Calderón, P. (2000). Microbiología Alimentaria : Metodología Analítica para Alimentos y Bebidas. Madrid: Ediciones Díaz de Santos. Recuperado de https://es.scribd.com/document/339713504/Microbiologia-alimentaria-metodologiaanalitica-para-alimento-pdf

Pundir, R. K., \& Jain, P. (2010). Antimicrobial Activity of Allium sativum Ethanolic Extract against Food Associated Bacteria and Fungi. Drug Invention Today, 2(4), 229-232.

Robledo, M. de L., Marín, S., \& Ramos, A. J. (2001). Contaminación Natural con Micotoxinas en Maíz Forrajero y Granos de Café Verde en el Estado de Nayarit (México). Revista Iberoamericana de Micología, 18, 141-144.

Samuel, J. K., Andrews, B., \& Jebashree, H. S. (2000). In vitro Evaluation of the Antifungal Activity of Allium sativum Bulb Extract against Trichophyton rubrum, a Human Skin Pathogen. World Journal of Microbiology and Biotechnology, 16, 617-620. https://doi.org/10.1023/A:1008972016316

San Blas, G., Urbina, J. A., Marchán, E., Contreras, L. M., Sorais, F., \& San Blas, F. (1997). Inhibition of Paracoccidioides brasiliensis by Ajoene is Associated with Blockade of Phosphatidylcholine Biosynthesis. Microbiology, 143, 1583-1586.

Sánchez, E. (2002). Inhibición del Crecimiento y la Producción de Toxinas de Aspergillus flavus y Aspergillus parasiticus por Extractos de Plantas del Género Agave. Universidad Autónoma de Nuevo León.

Sandosskumar, R., Karthikeyan, M., Mathiyazhagan, S., Mohankumar, M., Chandrasekar, G., \& Velazhahan, R. (2007). Inhibition of Aspergillus flavus Growth and Detoxification of Aflatoxin B1 by the Medicinal Plant Zimmu (Allium sativum L. x Allium cepa L.). World Journal of Microbiology and Biotechnology, 23, 1007-1014. https://doi.org/10.1007/s11274-006-9327-x

Tagoe, D. N. ., Nyarko, H. D., \& Akpaka, R. (2011). A Comparison of the Antifungal Properties of Onion (Allium cepa), Ginger (Zingiber officinale) and Garlic (Allium sativum) against Aspergillus flavus, Aspergillus niger and Cladosporium herbarum. Medicinal Plant, 5(3), 281-287. https://doi.org/10.3923/rjmp.2011.281.287

Tequida, M., Cortez, M., Rosas, E. C., López, S., \& Corrales, C. (2002). Efecto de Extractos Alcohólicos de Plantas Silvestres Sobre la Inhibición de Crecimiento de Aspergillus flavus, Aspergillus niger, Penicillium Fusarium moniliforme y Fusarium poae. Revista Iberoamericana de Micología, 1(6), 84-88.

Torija, E., Matallana, C., \& Chalup, N. (2013). El Ajo y la Cebolla: de las Medicinas Antiguas al Interés Actual. Boletín de la Real Sociedad Española de Historia Natural Sección Biología, 107, 29-37.

Torres, J., \& Romero, H. (2012). Actividad Antifúngica In vitro del Ajoeno en Cinco Aislamientos Clínicos de Histoplasma capsulatum var. capsulatum. Revista Iberoamericana de Micologia, 29(1), 24-28. https://doi.org/10.1016/j.riam. 2011.04.001

Vega, V. (2012). Hongos Micotoxigénicos y Aflatoxinas en Granos de Maíz de Diferentes Orígenes Geográficos de la República Mexicana. Universidad Autónoma Agraria "Antonio Narro".

Villa, A., Pérez, R., Morales, H., Basurto, M., Soto, J., \& Martínez, E. (2014). Situación Actual en el Control de Fusarium spp. y Evaluación de la Actividad Antifúngica de Extractos Vegetales. Acta Agronómica, 64(2), 194-205. https://doi.org/10.15446/acag. v64n2.43358 
